\title{
Condições de trabalho e transtornos mentais comuns em trabal hadores da rede básica de saúde de Botucatu (SP)
}

\author{
Working conditions and common mental disorder \\ among primary health care workers from Botucatu, São Paulo State
}

Ludmila Candida de Braga ${ }^{1}$

Lidia Raquel deCarvalho ${ }^{2}$

M aria Cecília Pereira Binder ${ }^{3}$

${ }^{1}$ Universidade Estadual

Paulista Júlio de M esquita Filho. Av. Bento Lopes $s / n=$, Distrito de Rubião Jr. 18618-970 Botucatu SP. sts@btu.unesp.br

${ }^{2}$ Departamento de Bioestatística, Instituto de Biociências, Universidade Estadual Paulista Júlio de Mesquita Filho.

${ }^{3}$ Departamento deSaúde

Pública, Faculdadede

Medicina deBotucatu,

UniversidadeEstadual

Paulista Júlio de M esquita

Filho.
Abstract Common mental disorders (CMD) present high prevalence among general populations and workers with important individual and social consequences. This cross-sectional and de scriptive study explores the relationship between psychological job demands, job control degree and job support and prevalence of CM D among primary health care workers of Botucatu - SP. The data collection was carried out using an unidentified self-administered questionnaire, with emphasis on items relating to demand-control-support situation and occurrence of CM D (Self Reporting Questionnaire, SRQ-20). The data were stored using the software Excel / O ffice XP 2003, and the statistical analyses were performed in SAS system. It was evidenced that $42.6 \%$ of primary health care workers presented CM D. The observed association - high prevalence of CM D with high-strain job (Karasek model) and low prevalence of CM D with low-strain job - indicates that, in the studied city, primary health care work conditions are contributive factors to workers' illness. The survey reveals the need of interventions aiming at caring the workers and also gets better work conditions and increase social support at work.

Key words Primary health care, Demand-control model, Occupational health, M ental health, M ental disorders
Resumo Ostranstornos mentais comuns (TM C) apresentam elevada prevalência em populações gerais e de trabalhadores, com consequências individuais e sociais importantes. Este estudo, transversal e descritivo, explora a relação entre demandas psicológicas, grau de controlee presença de suporte social no trabalho e prevalência de TM C em trabalhadores da rede básica de saúde de Botucatu (SP). A coleta de dados foi feita por meio de questionário autoaplicável, não identificado, com destaque para itens relativos à demanda-controlesuportee presença de TM C (Self Reporting Questionnaire, SRQ -20). As informações foram inseridas em banco de dados construído com Excel/Office XP 2003 e a análise estatística, efetuada com o programa SAS. Constatou-se que $42,6 \%$ dos trabalhadores apresentavam TM C. A observação de associação - alta prevalência de TM C com elevado desgaste (classificação de Karasek) e baixa prevalência de TM C com baixo desgaste- indica que, no município estudado, as condições de trabalho na atenção básica constituem fator contributivo não negligenciável ao adoecimento dos trabalhadores. Revela-se a necessidade de intervenções direcionadas ao cuidado aos trabalhadores, melhoria das condições de trabal ho eaumento do suporte social no trabalho.

Palavras-chave Atenção primária à saúde, M odelo demanda-controle, Saúdedo trabalhador, Saúde mental, Transtornos mentais 
Introdução

Em princípio, o trabalho deveriaser fontedeprazer, já que, através dele, o homem se constitui sujeito e reconhece sua importância para a sobrevivência de outros seres humanos. A Carta de Ottawa ${ }^{1}$, elaborada em 1986, na cidade canadense de mesmo nome, durante a Primeira Conferência Internacional sobre Promoção da Saúde, reconhece o trabal ho como fonte de saúde para o homem.

Para Leplat ${ }^{2}$, no desenvolvimento do trabaIho, além de modificar a natureza, o homem modifica-se a si próprio, na medida em que 0 trabalho propicia ou cerceia o desenvolvimento de suas potencialidades latentes. 0 autor enfatiza a importância da natureza do trabalho e das condições em que é exercido no desenvolvimento das capacidades dos trabalhadores.

Entretanto, ao longo da história, o trabalho, para a maioria da humanidade, tem representado dor, adoecimento e morte, fruto das diferentes formas de exploração a que os homens têm sido submetidos ao longo dos séculos e que, nos primórdios do século XXI, têm se intensificado.

Praticamente em todos os países do mundo, vêm ocorrendo profundas transformações no mundo do trabal ho que, por um lado, guardam estreitas relações com a crise do capital ismo iniciada nos anos setenta e, por outro, com as notáveis inovações tecnológicas, particularmente nos campos da informática, da automação e das comunicações, a ponto de ser denominada terceira revolução industrial ${ }^{3}$.

A mais recente crise do capitalismo torna-se evidente quando os países capitalistas centrais começam a ser atingidos por prolongada queda nas taxas de acumulação, o que leva o capital a buscar alternativas para retornar aos níveis precedentes, processo que, segundo Antunes ${ }^{3}$, vem sendo conhecido como acumulação flexível. Para este autor, isso tem significado aumento das taxas de exploração da classe-que vive-do-trabaIho, com sérias consequências para a qualidade de vida e para a saúde e segurança dos trabal hadores. Kurz ${ }^{4}$ enfatiza os efeitos dessa realidade: intensificação do trabalho, aumento da jornada, desregulamentação de direitos trabal histas eprecarização do trabalho.

Com alguns anos de defasagem, essas mudanças no mundo do trabalho começaram a chegar ao Brasil, com efeitos mais dramáticos, uma vez que o "estado de bem-estar social", conquistado nos países desenvolvidos, no caso brasilei ro estava longe de ser alcançado. E, além dis- so, o país estava mergulhado, há décadas, em crise econômica.

Segundo Pochmann $n^{5}$, no contexto da concorrência internacional não regulamentada, a concentração da inovação tecnológica nas grandes corporações agravou a exclusão de muitos países do espaço econômico. 0 corolário disto tem sido o aumento do desemprego e o predomínio da criação de empregos precários, com baixos salários, acarretando retrocesso produtivo e social para o país.

$\mathrm{N}$ as últimas décadas, tem-se observado aumento do número de trabalhadores sem proteção social: empregos precários, organização de falsas cooperativas, admissão em falsos estágios, exercício de atividades como falsos autônomos ou em situação de falsas terceirizações ${ }^{5}$.

As influências do trabalho sobre a saúde dos trabal hadores são conhecidas desde a antiguidade e, ao longo dos últimos três séculos, cresceu progressivamente a compreensão das relações entre trabalho e processo saúde-doença. Avanços em vários campos do conhecimento têm contribuído para isto, particularmente os da epidemiologia, da psicologia social, da ergonomia, dentre outros ${ }^{6}$.

A precarização do trabal ho tem sido responsável pela piora das condições de saúde e pela mudança do perfil epidemiológico de adoecimento dos trabalhadores, com destaque para 0 aumento das doenças relacionadas ao trabalho ou atípicas e, entre elas, dos transtornos mentais ${ }^{6}$.

Transtornos mentais comuns (TMC) é uma expressão criada por Goldberg e Huxley ${ }^{7}$ para designar sintomas tais como insônia, fadiga, irritabilidade, esquecimento, dificuldade de concentração e queixas somáticas, que demonstram ruptura do funcionamento normal do indivíduo, mas não configuram categoria nosológica da $10^{\underline{a}}$ Classificação Internacional deD oenças (CID-10), bem como dos $\mathrm{M}$ anuais de Diagnóstico e Estatística (DSM) da Associação Psiquiátrica Americana ${ }^{8}$. Entretanto, os transtornos mentais comuns constituem problema de saúde pública e apresentam impactos econômicos relevantes em função das demandas geradas aos serviços de saúde e do absenteísmo no trabalho ${ }^{8}$.

No Brasil, vários autores têm revelado alta prevalência desses transtornos nas populações estudadas ${ }^{9-13}$, cujas consequências, individuais e sociais, reforçam a necessidade de identificação precoce, para orientar interven ções individuaise coletivas $^{14,15}$.

Em vários estudos, numerosos fatores têm sido associados à prevalência detranstornosmen- 
tais comuns, dentre os quais atributos do indivíduo $0^{10,11,16,17}$, aspectos sociais e familiares, ${ }^{9,13,17-20} \mathrm{e}$ aspectos do trabalho. Dentre os últimos, cabe citar a baixa renda ${ }^{11,16}$, a exclusão do mercado formal de trabalho ${ }^{10,16,18}$ e o desemprego ${ }^{18} \mathrm{e}$, em destaque, as altas demandas psicológicas no trabaIho e o baixo controle sobre o trabalho ${ }^{12}$.

Segundo Karasek ${ }^{21}$, as demandas são as pressões psicológicas a que os trabal hadores são submetidos no trabalho e podem originar-se da quantidade detrabal ho a executar na unidade de tempo e/ou do descompasso entre as capacidades do trabal hador e o trabalho a executar. Quanto ao controle, trata-se do grau de autonomia ou possibilidade que ele tem de "governar" o seu trabal ho, a partir de suas habilidades e conhecimentos. Esse autor desenvolveu modelo de análise do trabalho denominado demanda-controle, cuja representação se dá em uma figura quadrangular, na qual cada quadrante representa associações entre os níveis das demandas e os graus de controle, ou seja:

- demandas psicológicas el evadas ebaixo controle do trabalho ou job strain, configurando condição de trabalho geradora de desgaste psicológico el evado;

- demandas psicológicas elevadas e alto grau de controle sobre o trabalho ou trabalho ativo, situação considerada como potencialmente de safiadora e capaz de propiciar o desenvolvimento das potencialidades dos trabalhadores;

- baixas demandas psicológicas e baixo controle ou trabalho passivo, configurando situações desestimulantes, geradoras de tédio e de desinteresse;

- baixas demandas psicológicas eal to grau de controle ou baixo desgaste, situação em que haveria pouco desgaste para o trabalhador, uma vez que ele tem as melhores condições para planejar e para executar seu trabalho.

A esse modelo bidimensional, Johnson ${ }^{22}$ acrescentou o suporte social, considerado uma terceira dimensão.

A saúde mental dos profissionais de saúde: o caso da aten ção básica

Segundo o M inistério da Saúde ${ }^{23}$, a aten ção básica constitui um conjunto de ações de saúde, no âmbito individual e coletivo, que abrangem a promoção ea proteção da saúde, a prevenção de agravos, o diagnóstico, o tratamento, a reabilitação e a manutenção da saúde. É desenvolvida por meio do exercício de práticas gerenciais e sanitárias demo- cráticas e partici pativas, sob forma de trabalho em equipe, dirigidas a populações de territórios bem delimitados, pelas quais assumea responsabilidade sanitária, considerando a dinamicidade existente no território em que vivem essas populações. Utiliza tecnologias de elevada complexidade e baixa densidade, que devem resolver osproblemas desaúde de maior frequência e relevância em seu território. É o contato preferencial dos usuários com os sistemas de saúde. Orienta-se pelos princípios da universalidade, da acessi bilidadee da coordenação do cuidado, do vínculo e continuidade, da integralidade, da responsabilização, da humanização, da equidade e da participação social.

Cunha ${ }^{24}$ ressalta a complexidade da atenção básica, que implica exigências diversificadas aos trabal hadores, que não se esgotam na realização do diagnóstico e na orientação do tratamento. Ao contrário, a atenção básica busca superar a visão reducionista denecessidades de saúde como equivalentes a processos fisiopatológicos, transformando-se em espaço privilegiado para a prática da integralidade do cuidado e para a valorização da subjetividade ${ }^{25}$.

Além das exigências inerentes à atenção integral à saúde eà humanização das práticas, os trabalhadores da saúde ficam expostos a situações geradas pela pobreza e pelas desigualdades sociais, assim como pelas deficiências dos demais níveis do sistema de saúde. E mais, necessitam desenvolver múltiplas habilidades eincorporar tecnologias bastante complexas para superar os desafios que se colocam no cotidiano do trabalho.

A esses aspectos somam-se as mudanças atuais no mundo do trabalho, que não pouparam o setor saúde da precarização, gerando medo, isolamento e submissão dos trabalhadores. A falta de reconhecimento profissional, aliada à discussão tardia sobre planos de cargos e salários para os trabalhadores do Sistema Único de Saúde (SUS) e ao arrocho salarial no âmbito do sistema de saúde, dentre outros, são reveladores dessa precarização.

Em relação aos usuários do SUS, verifica-seo aumento da demanda por serviços públicos de saúde, de tal sorte que cerca de $75 \%$ da população brasileira tornou-se quase inteiramente dependente desse sistema ${ }^{26}$. Para Luz ${ }^{27}, 0$ aumento da busca por cuidados em saúde constitui uma espécie de compensação pelo esvaziamento de valores culturais da sociedade, dilacerados pelo capitalismo.

A associação de transtornos psíquicos às profissões de saúde tem sido estabelecida por uma série deestudosque, atéo momento, têm privile- 
giado as categorias dos médicos e dos enfermeiros, sobretudo em atividades hospitalares, particularmente deurgência/emergênciaecentros de terapia intensiva ${ }^{12,28-30}$.

Alguns estudos têm revelado frequência elevada de transtornos mentais comuns entre os profissionais investigados ${ }^{12,30}$, associada a situações de risco, conforme modelo demanda-controle, especialmente as com altas demandas.

Abordando a relação trabalho-saúde entre enfermeiros do Programa de Saúde da Família em parte da grande São Paulo, Santos ${ }^{31}$ identifica, no cotidiano do trabalho desses profissionais, situações de alta exigência emocional, acarretando desgaste físico e mental.

Este estudo, cuja motivação inicial relacionou-seestreitamentecom a vivência profissional de duas das autoras, teve como objetivo explorar as relações entre demandas psicológicas, grau de controle e presença de suporte social no trabalho e prevalência de transtornos mentais comuns (TMC) em trabalhadores da rede básica de saúde de Botucatu (SP).

\section{M etodologia}

Trata-se de estudo populacional de corte transversal, realizado deabril a outubro de 2006, em município de médio porte do Estado de São Paulo.

Fundada em 23 de dezembro de 1843, Botucatu - Ibytu-katu, que em tupi significa "bons ares" - éuma cidade paulista de 121.274 habitantes $^{32}$, localizada a $224,8 \mathrm{~km}$ da capital. 0 perfil de atividade econômica do município caracterizase por participação nos setores de prestação de serviços e industrial ${ }^{33}$.

A rede básica de saúde do município compõe-se de dezessete unidades de saúde (oito unidades de saúde da família, oi to centros de saúde - dois dos quais gerenciados pela Faculdade de M edicina de Botucatu, Unesp - e um Centro de Referência em Saúde do Trabalhador).

Até 1986, a cidade contava com duas unidades básicas de saúde, então denominadas centros de saúde. N este ano, portanto antes da implantação do SUS, teve início a expansão desses serviços, com a criação de unidades municipais. Em 2004, foram implantados o Centro de Refe rência em Saúde do Trabalhador e o Programa deSaúdeda Família, esteúltimo priorizando áreas periféricas ecom população em piores condições de vida e de saúde.

\section{Casuística}

No período estudado, a rede básica de saúde possuía efetivo de 399 trabalhadores, dos quais 378 aderiram ao estudo. Dezenove trabalhadores afastados por motivo de doença foram incluídos no estudo, com intuito de reduzir o efeito do trabalhador sadio ${ }^{34}$.

Participaram do estudo todas as categorias profissionais que compõem o efetivo da rede básica: trabalhadores administrativos, agentes comunitários, auxiliares/técnicos deenfermagem, auxiliares de consultório dentário, auxiliares de serviços gerais, enfermeiros, médicos, outros profissionais de nível superior eoutros profissionais denível médio.

\section{Coleta deinformações}

Foi realizada por meio de questionário autoaplicável, não identificado, distribuído e recolhido por profissional habilitada para fornecer as explicações necessárias aos trabalhadores.

O questionário compôs-se de quatro seções: Seção A: informações sociodemográficas dos trabalhadores (idade, sexo, situação conjugal, naturalidade, escolaridadeeexistência dedependentes);

Seção B: informações sobre o trabalho (vínculo empregatício, ocupação, atividadeexercida, características da jornada de trabalho, etc.);

Seção C: questões para avaliar a satisfação no trabalho (objeto de outro estudo);

Seção D: questões destinadas à exploração da situação de trabalho relativas às demandas psicológicas, ao grau de controle sobre o próprio trabalho e à existência de apoio social no trabaIho. Questões destinadas à investigação de TM C, por meio do Self Reporting Questionnaire ou SRQ-20.

Para avaliação dos aspectos demanda/controle/suporte, utilizou-sea versão resumida do questionário de Karasek, conhecido como Job Stress Scale, traduzido e validado por Alves et al..$^{35}$ para utilização no Brasil. Trata-se de dezessete questões, das quais cinco referem-se às demandas do trabalho, seis, ao grau de controle exercido pelo trabal hador sobre sua atividade, e seis, aos mecanismos de suporte no trabal ho existentes.

0 aspecto demanda tinha como mínimo e máximo possíveis, respectivamente, 5 e 20 pontos, o controle, 6 e 24 pontos e o suporte (apoio social), 6 e 24 pontos. Foram criadas duas categorias para cada aspecto, "alto" e "baixo", utili- 
zando-se como ponto de corte 12,5 para demanda e 15 para controle e para suporte.

A identificação de transtornos mentais comuns foi efetuada por meio do Self Reporting Questionnaire - SRQ-20, desenvolvido por $\mathrm{Har}$ ding et al. ${ }^{36}$, sob coordenação da Organização Mundial de Saúde e validado para utilização no Brasil por M ari e Willians ${ }^{37}$, tendo sido observadas sensibilidade de $83 \%$ eespecificidade de $80 \%$. Estudos internacionais encontraram sensibilidade e especificidade variando de $62,9 \%$ a $99 \%$ e $44 \%$ a $95 \%$, respectivamente ${ }^{38}$. Estudo realizado em Pernambuco determinou sensibilidade de $62,0 \%$ e especificidade de $80,0 \%{ }^{16}$.

A versão mais utilizada em estudos de base populacional é composta por vinte questões: quatro sobre sintomas físicos e dezesseis sobre sintomas psicoemocionais (SRQ-20) ${ }^{9,13,16}$. Asrespostas são do tipo "sim" ou "não", atribuindose, respectivamente, valores de 1 e 0 . Os pontos de corte sugeridos por Mari e Willians são $5 / 6$ para homens e $7 / 8$ para mulheres, pois, no estudo desses autores, o valor preditivo positivo foi menor entre os homens ( $66 \%)$ do que entre as mulheres (83\%).

Neste estudo, foram adotados os pontos de corte sugeridos por M ari e Willians ${ }^{37}$.

\section{Procedimentos prévios}

0 passo inicial constituiu-se na apresentação do projeto ao Comitê de Ética em Pesquisa da Faculdade de M edicina de Botucatu - Unesp, onde foi analisado e aprovado (processo $n^{\circ} 559 / 2004$ ). Em seguida, obteve-se, junto à Secretaria Municipal deSaúde, a relação detrabal hadores da rede básica segundo a função exercida e a unidade em quetrabal hava, informações que foram confrontadas com as existentes nos serviços.

A primeira autora, então, visitou todas as unidades de saúde para explicar aos trabalhadores os objetivos do estudo e como este seria conduzido, ocasião em que os que concordaram em participar preencheram o termo deconsentimento livree esclarecido.

Visando verificar o tempo aproximado de preenchimento e a clareza do instrumento de coleta de informações, foi realizado piloto em unidade básica de saúde deum município vizinho.

\section{Aplicação dos questionários}

Os trabal hadores que aderiram ao estudo e que estavam em atividade preencheram o questionário no próprio local de trabalho e os afastados, nas respectivas residências ou em outro local indicado por eles.

Os questionários foram distribuídos e coletados no mesmo dia por uma mesma profissional (entrevistadora), com experiência nesse tipo de atividade. Sempre que um trabalhador, por razões relacionadas à dinâmica do serviço, não podia interromper suas atividades para preencher o questionário, a entrevistadora, de comum acordo com o trabalhador e a chefia do serviço, voltava em outra ocasião, a fim de evitar transtorno ao serviço, bem como restringir perda de sujeitos que haviam aderido à pesquisa.

Antes da entrega dos questionários, a entrevistadora explicava novamente os objetivos do estudo, dava as instruções gerais sobre o preenchimento, aguardando sua devolução. No caso dos trabalhadores afastados, a entrevistadora, após contato telefônico e autorização do trabalhador, dirigia-se a sua residência ou outro local indicado por ele para a aplicação do questionário.

Os questionários eram identificados apenas quanto à unidade de saúde. Durante a coleta de informações, entrevistadora e a autora principal reuniram-se semanalmente para entrega e revisão dos questionários.

\section{Construção do banco de dados}

Foram construídos dois bancos de dados para confrontar as informações eidentificar possíveis erros de digitação. Foram utilizados os programas Excel, do O ffice XP 2003, e Epi Info 6.0.

\section{Análisedos dados}

Para detectar a existência de associações entre as variáveis estudadas, utilizou-se o teste do quiquadrado, tendo-se estabelecido 5\% como nível designificância.

Nos cruzamentos em que foram detectadas associações entre as variáveis estudadas, aplicouse 0 teste de Goodman, utilizando-se letras minúsculas eletras maiúsculas, respectivamente, ao lado e abaixo das porcentagens. Estas, quando acompanhadas por letras iguais, indicam que as porcentagens não diferem significativamenteentresi. As letras minúsculas comparam os valores 
nas linhas e as maiúsculas, nas colunas das tabelas. $\mathrm{Na}$ análise estatística, foi utilizado o pacote estatístico SAS.

\section{Resultados}

No período do estudo, as dezessete unidades de saúde possuíam 399 trabal hadores, dos quais dezenoveestavam em licença por motivo de saúde. Do total, $378(94,7 \%)$ aderiram ao estudo e responderam os questionários. Destes, 366 trabalhadores estavam em atividade e doze, afastados por motivo de saúde.

Em relação aos principais atributos pessoais, sabe-se que $85 \%$ dos indivíduos possuíam idades de 20 a 59 anos; $80 \%$ eram mulheres; $58 \%$ viviam em união estável; $54 \%$ possuíam nível médio de escolaridadee $44 \%$, nível superior; $59 \%$ eram naturais de Botucatu ou de cidades vizinhas e $65 \%$ possuíam algum dependente.

Situação de trabalho avaliada pelo questionário demanda/controle/suporte

D os 378 indivíduos que compuseram a casuística, vintenão preencheram todosositens do questionário demanda-controle-suporte e foram excluídos da análise quanto à situação de trabal ho avaliada por estas questões.

Os resultados obtidos para os 358 trabal hadores que responderam todas as questões encontram-se na Figura 1. Esta figura revela predomínio desituação de trabalho ativo com 53,1\% dos trabalhadores. Os números representados na figura possibilitam calcular o número de trabalhadores submetidos à situação de demandas psicológicas el evadas- 263 indivíduos ou 73,5\%.

\section{Ocorrência detranstornos mentais comuns}

Dos 378 trabalhadores que aderiram ao estudo, $161(42,6 \%)$ apresentaram TM C. Não foram observadas associações entre ocorrência destes transtornos e fatores sociodemográficos como gênero, idade, escolaridade, situação conjugal, naturalidadee existência de dependentes diretos do trabalhador.

A distribuição dos trabalhadores segundo ocorrência de TM C e categoria profissional encontra-se na Tabela 1, não tendo sido observadas diferenças estatisticamente significantes entre as várias categorias profissionais. Entretanto,

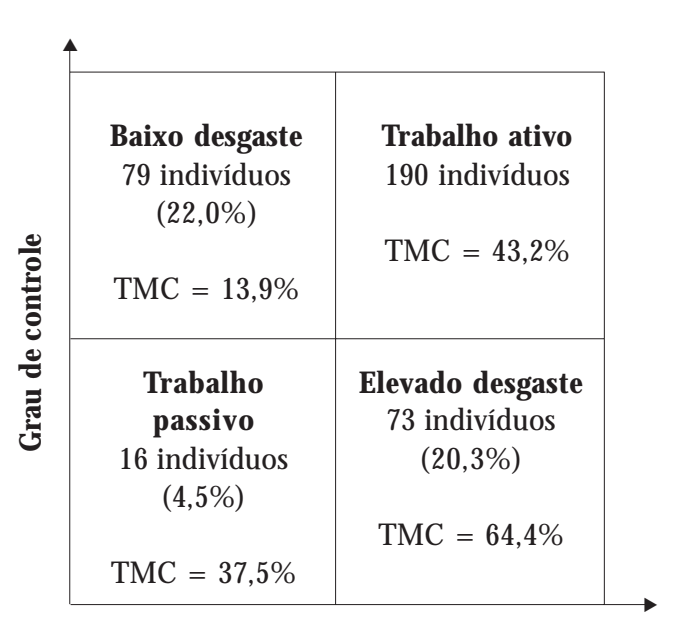

Demandas psicológicas

Figura 1. Distribuição dos trabalhadores da rede básica de saúde segundo situação de trabalho avaliada pelo modelo de Karasek e presença de transtorno mental comum (TM C). Botucatu, 2007.

considerando-se apenas os dois grandes grupos - trabalhadores em atividades que exigem nível médio de escolaridade e trabal hadores em atividades que exigem escolaridade superior - , constatou-seque, respectivamente, $46,5 \%$ e $34,1 \%$ dos profissionais apresentaram TM C. 0 teste do quiquadrado revelou associação de ocorrência de TM C entre os dois grupos e ocorrência de TM C. $O$ teste de Goodman mostrou que a diferença observada era estatisticamente significante.

Não foi observada associação estatisticamente significativa quanto à ocorrência de TM C entre as dezessete unidades de saúde. Entretanto, foi possível identificar três grupos distintos de unidades quanto à ocorrência desses transtornos (Tabela 2). 0 teste de Goodman revelou que a diferença observada na proporção de casos de TM C nas unidades básicas de saúde do grupo 1 e do grupo 3 foi estatisticamente significante. Já entre 0 grupo 1 e o grupo 2, assim como entre este e o grupo 3, as diferen ças não foram estatisticamentesignificantes.

A distribuição dos portadores de TMC segundo as quatro situações possíveis do modelo de Karasek (demanda-controle) encontra-se na Figura 1 e, mais detal hadamente, na Tabela 3, na qual a aplicação do teste do qui-quadrado revelou associação estatisticamente significante 
$(p<0,0001)$ entre algumas situações de trabalho epresençadeTM C. $O$ testede Goodman indicou que a ocorrência de TM C foi significativamente mais elevada na situação de el evado desgaste $(64,4 \%)$ em comparação com as demais e significativamentemenor ( $13,9 \%)$ na situação de baixo desgaste.

A influência do suporte social no trabalho como fator de proteção em relação à ocorrência de TMC nas diferentes situações de trabalho do ponto de vista das demandas psicológicas e do grau de controle do trabalhador sobre seu trabalho pode ser analisada na Tabela 4. A aplicação do teste do qui-quadrado mostrou existência de associação $(p<0,0001)$ e os resultados do teste de Goodman revelaram que algumas diferenças foram estatisticamente significantes e outras não (Tabela 4).

Tabela 1. Distribuição dos trabalhadores da rede básica de saúde, segundo categoria profissional e presença de transtornos mentais comuns. Botucatu, 2007.

\begin{tabular}{|c|c|c|c|}
\hline \multirow[b]{2}{*}{ Categoria profissional } & \multicolumn{2}{|c|}{ Transtorno mental comum } & \multirow[b]{2}{*}{$\begin{array}{c}\text { Total } \\
\mathrm{N} \cong(\%)\end{array}$} \\
\hline & $\begin{array}{l}\text { Presente } \\
\mathrm{N} \cong(\%)\end{array}$ & $\begin{array}{l}\text { Ausente } \\
\mathrm{N} \cong(\%)\end{array}$ & \\
\hline $\begin{array}{l}\text { Com exigência de nível médio de } \\
\text { escolaridade }\end{array}$ & $\begin{array}{r}118(46,5) a \\
A\end{array}$ & $\begin{array}{r}136(53,5) \text { a } \\
\text { B }\end{array}$ & $254(100,0)$ \\
\hline Auxiliar de serviços gerais & $15(55,6)$ & $12(44,4)$ & $27(100,0)$ \\
\hline Agente comunitário & $36(50,7)$ & $35(49,3)$ & $71(100,0)$ \\
\hline Auxiliar de consultório dentário & $8(47,1)$ & $9(52,9)$ & $17(100,0)$ \\
\hline Auxiliar de enfermagem & $46(43,8)$ & $59(56,2)$ & $105(100,0)$ \\
\hline Auxiliar administrativo & $11(39,3)$ & $17(60,7)$ & $28(100,0)$ \\
\hline Outros profissionais de nível médio & $2(33,3)$ & $4(66,7)$ & $6(100,0)$ \\
\hline $\begin{array}{l}\text { Com exigência de nível superior de } \\
\text { escolaridade }\end{array}$ & $\begin{array}{r}42(34,1) b \\
B\end{array}$ & $81(65,9)$ a & $123(100,0)$ \\
\hline Enfermeiro & $12(48,0)$ & $13(52,0)$ & $25(100,0)$ \\
\hline M édico & $18(33,3)$ & $36(66,7)$ & $54(100,0)$ \\
\hline Outras profissões & $12(27,3)$ & $32(72,7)$ & $44(100,0)$ \\
\hline Sem informação & $1(100,0)$ & - & $1(100,0)$ \\
\hline Total & $161(42,6)$ & $217(57,4)$ & $378(100,0)$ \\
\hline
\end{tabular}

Teste deGoodman - letras maiúsculas (A,B) comparam colunas eminúsculas (a,b), linhas.

Tabela 2. Distribuição dos trabalhadores da rede básica de saúde, segundo presença de transtornos mentais comuns e grupos de unidades de saúde. Botucatu, 2007.

\begin{tabular}{|c|c|c|c|c|c|c|}
\hline \multirow{3}{*}{ Grupos de Unidades de Saúde } & \multicolumn{4}{|c|}{ Transtorno mental comum } & & \\
\hline & \multicolumn{2}{|c|}{ Presente } & \multicolumn{2}{|c|}{ Ausente } & \multicolumn{2}{|c|}{ Total } \\
\hline & $\mathbf{N} 0$ & $\%$ & $\mathbf{N} 0$ & $\%$ & No & $\%$ \\
\hline $\begin{array}{l}\text { GRUPO } 1(A, I, J, G, L, N) \\
>=50,0 \%\end{array}$ & 58 & $\begin{array}{c}54,2 \mathrm{a} \\
\mathrm{A}\end{array}$ & 49 & $\begin{array}{c}45,8 \text { a } \\
B\end{array}$ & 107 & 100,0 \\
\hline $\begin{array}{l}\text { GRUPO } 2(D, H, F, E, B) \\
>=37,0 \% \text { e }<50,0 \%\end{array}$ & 53 & $\begin{array}{c}45,7 a \\
A B\end{array}$ & 63 & $\begin{array}{c}54,3 a \\
A B\end{array}$ & 116 & 100,0 \\
\hline $\begin{array}{l}\text { GRUPO } 3(Q, P, M, C, O, R) \\
<37,0 \%\end{array}$ & 50 & $\begin{array}{c}32,3 \mathrm{~b} \\
\mathrm{~B}\end{array}$ & 105 & $\begin{array}{c}67,7 \mathrm{a} \\
\mathrm{A}\end{array}$ & 155 & 100,0 \\
\hline Total & 161 & 42,6 & 217 & 57,4 & 378 & 100,0 \\
\hline
\end{tabular}

Qui-quadrado $p=0,0014$

Teste de Goodman - letras maiúsculas ( $A, B)$ comparam colunas e minúsculas (a,b), linhas. 
Nas situações de trabal ho passivo e elevado desgaste, o suporte social no trabalho não influenciou de maneira estatisticamentesignificantea ocorrência de TM C. Na situação de elevado desgaste, verificou-se que, na existência de suporte social elevado no trabalho, a proporção de casos de TM C ( $29 / 49$ ou $59,2 \%)$ foi inferior à observada quando o suporte era baixo (18/24 ou $75 \%)$.

\section{Discussão}

A adesão ao estudo foi considera boa, apesar da perda de alguns questionários pel o preenchimento incompleto, particularmente das questões que avaliam a situação de trabal ho segundo o modelo de Karasek.

Tabela 3. Distribuição dos trabalhadores da rede básica de saúde, segundo situação em relação às exigências e ao controle no trabalho e presença de transtornos mentais comuns. Botucatu, 2007.

\begin{tabular}{|c|c|c|c|c|c|c|}
\hline \multirow{3}{*}{$\begin{array}{l}\text { Situação em relação à } \\
\text { demanda/controle }\end{array}$} & \multicolumn{4}{|c|}{ Transtorno mental comum } & & \\
\hline & \multicolumn{2}{|c|}{ Presente } & \multicolumn{2}{|c|}{ Ausente } & \multicolumn{2}{|c|}{ Total } \\
\hline & $\mathbf{N} 0$ & $\%$ & $\mathrm{~N} 9$ & $\%$ & No & $\%$ \\
\hline Desgaste elevado & 47 & $\begin{array}{c}64,4 \text { a } \\
\text { A }\end{array}$ & 26 & $\begin{array}{c}35,6 \mathrm{~b} \\
\mathrm{C}\end{array}$ & 73 & 100,0 \\
\hline Trabalho ativo & 82 & $\begin{array}{c}43,2 \mathrm{a} \\
\mathrm{B}\end{array}$ & 108 & $\begin{array}{c}56,8 \text { a } \\
\text { BC }\end{array}$ & 190 & 100,0 \\
\hline Trabalho passivo & 6 & $\begin{array}{c}37,5 \text { a } \\
\text { BC }\end{array}$ & 10 & $\begin{array}{c}62,5 \text { a } \\
\text { B }\end{array}$ & 16 & 100,0 \\
\hline Baixo desgaste & 11 & $\begin{array}{c}13,9 b \\
C\end{array}$ & 68 & 86,1 & 79 & 100,0 \\
\hline Total & 146 & 40,8 & 212 & 59,2 & $358 *$ & 100,0 \\
\hline
\end{tabular}

* Excluídos 20 trabal hadores que não preencheram todos os itens do instrumento de avaliação de demanda/ controle/ suporte. Qui-quadrado, $p<0,0001$

TestedeGoodman - letras maiúsculas $(A, B, C)$ comparam colunas eminúsculas $(a, b, c)$, linhas.

Tabela 4. Distribuição dos trabalhadores da rede básica de saúde, segundo situação de trabalho (demandacontrole), existência de suporte social no trabalho e ocorrência de transtornos mentais comuns. Botucatu, 2007.

\begin{tabular}{|c|c|c|c|c|c|}
\hline \multirow{3}{*}{ Situação de trabalho } & \multicolumn{4}{|c|}{ Suporte social } & \multirow{3}{*}{ Total } \\
\hline & \multicolumn{2}{|c|}{ Elevado } & \multicolumn{2}{|c|}{ Baixo } & \\
\hline & $\begin{array}{l}\text { TMC + } \\
\mathrm{N}^{\circ}(\%)\end{array}$ & $\begin{array}{l}\text { TMC - } \\
\mathrm{N}^{\circ}(\%)\end{array}$ & $\begin{array}{c}\mathrm{TMC}+\mathrm{N}^{\circ} \\
(\%)\end{array}$ & $\begin{array}{l}\text { TMC - } \\
\mathrm{N}^{\circ}(\%)\end{array}$ & \\
\hline Trabalho passivo & $\begin{array}{c}5(31,3) a b \\
A B\end{array}$ & $\begin{array}{c}10(62,5) a \\
A B\end{array}$ & $\begin{array}{c}1(6,2) b \\
A B\end{array}$ & $\begin{array}{c}-b \\
B\end{array}$ & $16(100,0)$ \\
\hline Trabalho ativo & $\begin{array}{c}65(34,2) a \\
A\end{array}$ & $\begin{array}{c}98(51,6) a \\
B\end{array}$ & $\begin{array}{c}17(8,9) b \\
A\end{array}$ & $\begin{array}{c}10(5,2) b \\
A\end{array}$ & $190(100,0)$ \\
\hline Desgaste baixo & $\begin{array}{c}11(13,9) b \\
B\end{array}$ & $\begin{array}{c}68(86,1) a \\
A\end{array}$ & $\begin{array}{l}-C \\
B\end{array}$ & $\begin{array}{l}-C \\
B\end{array}$ & $79(100,0)$ \\
\hline Desgaste elevado & $\begin{array}{c}29(39,7) a \\
A\end{array}$ & $\begin{array}{c}20(27,4) b \\
C\end{array}$ & $\begin{array}{c}18(24,7) a b \\
A\end{array}$ & $\begin{array}{c}6(8,2) b \\
A\end{array}$ & $73(100,0)$ \\
\hline Total & $110(30,7)$ & $196(54,7)$ & $36(10,1)$ & $16(4,5)$ & $358^{*}(100,0)$ \\
\hline
\end{tabular}

*Excluídos 20 trabalhadores que não preencheram todos os itens do instrumento de avaliação de demanda/ controle/ suporte. Qui-quadrado, $p<0,0001$

TestedeGoodman - letras maiúsculas $(A, B, C)$ comparam colunas eminúsculas $(a, b, c)$, linhas. 
A constatação de que a maioria dos profissionais estava exposta a situações de al tas demandas psicológicas no trabalho está de acordo com alguns estudos realizados entre profissionais de saúde ${ }^{30}$. Isso configura realidade preocupante, já que a alta demanda psicológica no trabalho predispõe ao adoecimento e, em alguns estudos, é descrita como a variável do modelo demandacontrole que melhor discrimina a ocorrência de TM C ${ }^{12,30}$

Algumas condições de trabal ho, como extensão da jornada de trabalho, tempo de locomoção trabal ho-casa-trabalho, fruição regular de férias, flexibilidade do horário detrabalho, realização de cursos de capacitação, possibilidade de informar à chefia sobre 0 andamento do trabalho e tratamento dado pela chefia às informações sobre o trabal ho, investigadas nesta mesma população, foram consideradas adequadas, porém os trabaIhadores revelaram-se insatisfeitos com a remuneração, o reconhecimento profissional e a impossibilidade de ascensão na carreira ${ }^{39}$.

A verificação de que os grupos submetidos a altas demandas psicológicas apresentam os maiores índices de baixo suporte no trabalho mostra combinação ruim, dada a presença de fator de risco para o adoecimento com ausência de fator protetor. M esmo assim, neste estudo, nas situações de trabalho passivo e el evado desgaste, o suporte não interferiu na ocorrência de TM C.

A ocorrência de TMC na população de trabal hadores da rede básica de Botucatu (42\%) foi aproximadamente duas vezes maior que a observada no estudo populacional - $21,7 \%$ (IC ${ }_{95 \%}$ $=19,2 \%-24,3 \%$ ) - efetuado por Lima $^{9}$ e maior também que as prevalências encontradas em outras populações ${ }^{10,17}$. Trata-se de resultado alarmante devido à magnitude do problema e ao impacto negativo dos transtornos mentais para os indivíduos e para a sociedade ${ }^{15}$.

A verificação de que a ocorrência de TMC não esteve associada aos fatores sociodemográficos abordados indica a necessidade de ampliar a investigação da associação a outros aspectos relativos tanto às condições de vida quanto de trabalho da população em questão.

Esperava-se encontrar diferenças estatisticamente significantes na ocorrência de TMC nas diferentes categorias profissionais, sinalizando possíveis peculiaridades dos processos de trabaIho como fatores estressores, fato não observado. M esmo assim, o estudo mostrou maior frequência de TMC no grupo de profissões com exigência de nível médio de escolaridade do que no grupo com exigência de nível superior, sem, contudo, fornecer elementos concretos que explicassem essa diferença. Cabeassinalar que, para todas as categorias, os valores encontrados para TMC foram superiores aos observados por Lima ${ }^{9}$ na população geral de Botucatu: $21,7 \%$ (IC ${ }_{95 \%} 19,2 \%-24,3 \%$ ).

A porcentagem de médicos $(33,3 \%)$ com TMC ficou abaixo da encontrada para o conjunto de trabalhadores $(42,6 \%)$. Apesar das diferenças não terem sido estatisticamente significantes, a posição de destaque na equipe de saúde e a possibilidade de ter outros vínculos de trabaIho e maior remuneração poderiam explicar ocorrência de menor número de casos de TM C entre os médicos.

Antes de afastar a existência de associação entre categoria profissional e desenvolvimento de TM C em trabal hadores da rede básica de saúde, énecessário dar continuidadeaos estudos das condições de vida e trabalho dessa população. Isto porque as categorias em que as porcentagens de portadores de TMC foram mais elevadas são as situadas em escalões hierárquicos inferiores, recebendo salários mais baixos e, em geral, submetidas a maiores exigências quanto ao cumprimento de horários.

A formação de três grupos de unidades com taxas de TM C semelhantes aponta a necessidade de se investir na identificação de características das unidades (condições de vida dos trabalhadores, população de abrangência, processo de trabalho, composição da equipe, características gerenciais, entreoutros) quepossam explicar essa semel hança e orientar possíveis intervenções visando à redução das taxas de adoecimento. N esta exploração inicial, não foram esclarecidas essas semelhanças.

A demonstração de que a ocorrência de TM C associou-se positivamente à situação de elevado desgastee, negativamente, à situação de baixo desgaste está de acordo com a literatura, ou seja, a situação de elevado desgaste apresenta maior risco à saúde e a de baixo desgaste, menor risco ${ }^{12,21,30,40}$. N esteestudo, a segunda maior prevalência deTM C foi observada nos trabalhadores classificados no grupo trabalho ativo, o quetambém vai ao encontro dos resultados de outros estudos ${ }^{12,30,40}$.

M esmo sem haver significância estatística, 0 achado de que em situação de elevado suporte social no trabal ho, a proporção de casos de TM C é inferior à observada quando o suporte é baixo está de acordo com os achados de Reis et al ${ }^{40} \mathrm{e}$ N ascimento Sobrinho et al. ${ }^{30}$. N as situações de trabalho ativo e de baixo desgaste, o suporte social no trabal ho influenciou demaneira estatisti- 
camente significante a ocorrência de TM C, concordando com os resultados obtidos por outros pesquisadores 30,40 .

É preciso assinalar que houve dispersão dos trabal hadores classificados nos grupos trabalho passivo eelevado desgaste, respectivamente, com dezesseis e 73 indivíduos, o que pode explicar a não observação de diferenças estatisticamente significativas entre os subgrupos com e sem suporte social no trabalho, ou seja, que o suporte social no trabalho não teria constituído, nesta casuística, fator de proteção para TMC.

De acordo com o modelo de Karasek, a maioria dos profissionais enquadrou-seem situações de trabalho caracterizadas por demandas psicológicas elevadas. A prevalência de TMC entre os trabal hadores da rede básica foi aproximadamente duas vezes superior à observada para a população geral de Botucatu. O bservou-se associação entre situações de trabalho caracterizadas por demandas psicológicas elevadas e ocorrência de
TM C. Para a maioria dos trabal hadores portadores de TM C, os sintomas assinalados no SRQ20 possuíam relação com o trabalho.

\section{Conclusões}

Este estudo demonstra que os profissionais da rede básica de saúde de Botucatu estão submetidos a demandas psicológicas elevadas no trabaIho. Além disso, evidenciam-se fatores de estresse no trabalho que implicam prevalência elevada de TM C entre esses trabalhadores. Tais resultados indicam necessidade deinterven ção, visando meIhorar as condições gerais de trabal ho e fornecer suporte social ao coletivo de trabalhadores.

É preciso atentar para o paradoxo representado por situações de trabalho que contribuem para 0 adoecimento de trabal hadores cujas atividades visam à promoção e à recuperação da saúde de outrem.

\section{Colaboradores}

LC Braga, LR Carvalho, M CP Binder participaram igualmente de todas as etapas da elaboração do artigo. 


\section{Referências}

1. Organização Pan-Americana da Saúde. Carta de Ottawa, Primeira Conferência Internacional para Promoção da Saúde. [site da Internet] 1986 [acessado 2007 mar 20]. Disponível em: http://www.org.br/ coletiva/uploadArq/Ottawa.pdf

2. Leplat J. Développement et dégradation des habilités dans le travail. In: Societé Française de Psychologie. Psychologie du travail. Équilibre ou fatigue par le travail? Paris: Entreprise M oderne d'Edition; 1980. p. 55-63.

3. Antunes R. Adeus ao trabalho? Ensaios sobre as me tamorfoses e a centralidade do mundo do trabalho. São Paulo: Cortez; 1995.

4. Kurz R. Absoluter mehrwert. [site da Internet] [acessado 2005 out 12]. Disponível em: htpp://www.exitonline.org

5. Pochmann M. O emprego na globalização. A nova divisão internacional do trabalho e os caminhos que 0 Brasil escolheu. São Paulo: Boitempo Editorial; 2001.

6. Brasil. M inistério da Saúde. Doenças relacionadas ao trabalho: manual de procedimentos para os serviços de saúde. Brasília: Ministério da Saúde; 2001.

7. Goldberg D, Huxley P. Common mental disorders a bio-social model. $2^{\text {nd }}$ ed. London: Tavistock/Routledge; 1993.

8. Coutinho ESF, Almeida-Filho N, Mari JJ. Fatores de risco para morbidade psiquiátrica menor: resultado de um estudo transversal em três áreas urbanas do Brasil. Rev Psiquiatr Clín 1999; 26:246-56.

9. Lima MCP. TMC e uso de álcool na população urbana de Botucatu - SP: um estudo de co-morbidade e utilização de serviços [tese]. São Paulo (SP): Faculdade de Medicina; 2004.

10. Ludermir $A B$. Inserção produtiva, gênero e saúde mental. Cad Saude Publica 2000; 16(3):647-659.

11. Costa JSD, Menezes AMB, Olinto M TO, Gigante $D P, M$ acedo $S$, Britto MAP, Fuchs SC. Prevalência de distúrbios psiquiátricos menores na cidade de Pelotas, RS. Rev Bras Epidemiol 2002; 5(2):164-173.

12. Araújo TM, Aquino E, M enezes $G$, Santos $C O$, Aguiar L. Aspectos psicossociais do trabalho e distúrbios psíquicos entre trabalhadoras de enfermagem. Rev. Saude Publica 2003; 37(4):424-433.

13. Costa AG, Ludermir AB. TMC e apoio social: estudo em comunidade rural da Zona da M ata de Pernambuco, Brasil. Cad Saude Publica 2005; 21(1):73-79.

14. Lima MS. Epidemiologia e impacto social. Rev Bras Psiquiatr 1999; 21:1-5.

15. Organização M undial da Saúde. Relatório mundial da saúde. Saúde mental: nova concepção, nova esperança. Lisboa: OM S; 2002.

16. Ludermir AB, M ello-Filho DA. Condições de vida e estrutura ocupacional associadas a TMC. Rev. Saude Publica 2002; 36(2):213-221.

17. Araújo TM, Pinho PS, Almeida MMG. Prevalência de TMC em mulheres e sua relação com as características sócio-demográficas e o trabalho doméstico. Rev. Bras. Saude Mater. Infant. 2005; 5(3):337-348.

18. Lima MS, Soares BGO, Mari JJ. Saúde e doença mental em Pelotas, RS: um estudo populacional. Rev Psiquiatr Calem 1999; 26(5):225-235.
19. Lopes CS, Faerstein E, Chor D. Eventos de vida produtores de estresse e TMC: resultados do Estudo Pró-Saúde. Cad Saúde Pública 2003; 19(6):17131720.

20. Volcan SM A, Sousa PLR, Mari JJ, Lessa B. Relação entre bem-estar espiritual e transtornos psíquicos menores: estudo transversal. Rev. Saude Publica 2003; 37(4):440-445.

21. Karasek R. [CD-ROM ]. Demand/control model: a social, emotional, and physiological approach to stress risk and active behaviour development. Geneva: International Labour Organization; 2005.

22. Johnson JV, Hall EM. Job strain, work place social support, and cardiovascular disease: a cross-sectional study of a random sample of the Swedish working population. Am Public Health 1988; 78(10):1336-1342.

23. Brasil. M inistério da Saúde. Política Nacional deAtenção Básica. Braślia: M inistério da Saúde; 2006.

24. Cunha GT. A construção da clínica ampliada na atenção básica. 2a ed. São Paulo: Hucitec; 2007.

25. Schraiber LB, M endes-Gonçalves RB. Necessidades de saúde e atenção primária. In: Schraiber LB, Nemes MIB, M endes- Gonçalves RB, organizadores. Saúde do adulto. Programas e ações na unidade básica. São Paulo: Hucitec; 2000. p. 29-47.

26. Bodstein R. Atenção básica na agenda da saúde. Cien Saude Colet 2002; 7(3):401-412.

27. Luz TM. Fragilidade social e busca de cuidado na sociedade civil de hoje. In: M attos RA, Pinheiro R, organizadores. Cuidado: as fronteiras da integralidade. Rio de Janeiro: Abrasco; 2005. p. 9-20.

28. Benevides-Pereira AMT. Burnout: quando o trabaIho ameaça o bem-estar do trabalhador. São Paulo: Casa do Psicólogo; 2002.

29. Seligmann E. Psicopatologia e saúde mental no trabalho. In: M endes R, organizador. Patologia do trabalho: atualizada e ampliada. São Paulo: Atheneu; 2003. p. 1141-1182.

30. Nascimento Sobrinho CL, Carvalho FM, Bonfim TAS, Cirino CAS, Ferreira IS. Condições de trabaIho e saúde mental dos médicos de Salvador, Bahia, Brasil. Cad Saude Publica 2006; 22(1):131-140.

31. Santos VC. A relação trabalho-saúde dos enfermeiros do PSF da região de Vila Prudente-Sapopemba: um estudo de caso [dissertação]. São Paulo (SP): Escola de Enfermagem; 2007.

32. Instituto Brasileiro de Geografia e Estatística. Cidades@. 2006 [site da Internet] [acessado 2007 mar 31]. Disponível em: http://www.ibge.gov.br/cidadesat/default.php

33. Fundação Sistema Estadual de Análise de Dados. Perfil M unicipal de Botucatu. 2006 [site da Internet] [acessado 2006 ago 8]. Disponível em: http:// www.seade.gov.br/produtos/perfil/perfil.php

34. Assenato G, Ferri G, Porro A, Corrado V. La metodologia epidemiologica in medicina del lavoro. In: Abrosi G, Foà V. Trattato di Medicina del Lavoro. Turim: Unione Tipografico-Editrice Torinese; 1996. p. 63-113. 
35. Alves MGM, Chor D, Faerstein E, Lopes CS, Werneck GL. Versão resumida da "job stress scale": adaptação para o português. Rev. Saude Publica 2004; 38(2):164-171.

36. Harding TW, de Arango M V, Baltazar J, Climent CE, Ibrahim H H, Ladrido-Ignacio L, M urthy RS, Wig NN. Mental disorders in primary health care: a study of their frequency and diagnosis in four developing countries. Psychol M ed 1980;10:231-241.

37. Mari JJ, Willians P. A validity study of a Psychiatric Screening Questionnaire (SRQ-20) in Primary care in the city of São Paulo. Br J Psychiatry 1986; 148:2326.

38. World Health Organization. A user's guide to the Self Reporting Questionnaire (SRQ). Geneva: Division of Mental Health; 1994.

39. Braga LC, Binder M CP. Condições de trabalho dos profissionais da rede básica de saúde de Botucatu SP. In: Anais do X Congresso Paulista de Saúde Pública. Saúde e Sociedade; 2007; Botucatu.

40. Reis EJFB, Carvalho FM, Araújo TM, Porto LA, Silvany Neto AM. Trabalho e distúrbios psíquicos em professores da rede municipal de Vitória da Conquista, Bahia, Brasil. Cad Saude Publica 2005; 21(5):1480-1490.

Artigo apresentado em 26/11/2007

Aprovado em 22/07/2008

Versão final apresentada em 06/08/2008 